LA-UR-96- $-\mathrm{p} 9$

Title:

\title{
Safe Extension of Red Blood Cell Storage Life
} at $4^{\circ} \mathrm{C}$

RECEIVED

APR 011996

OSTI

Submitted to:

\section{DOE Office of Scientific and Technical Information (OSTI)}

\section{Los Alamos}

NATIONAL LABORATORY

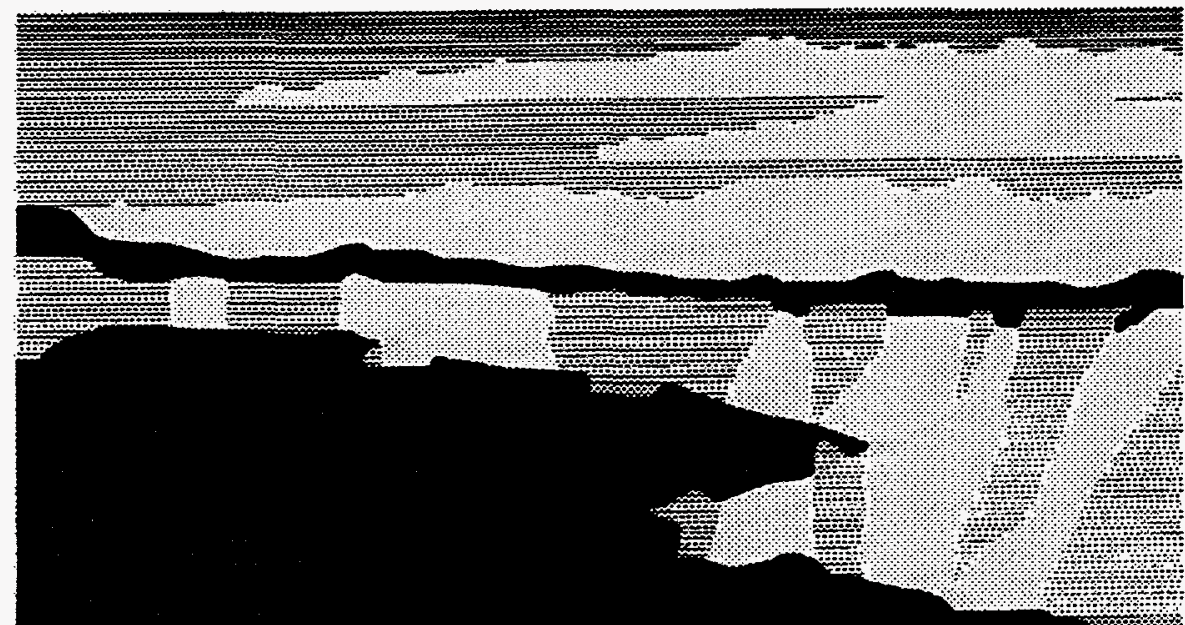

Los Alamos National Laboratory, an affirmative action/equal opportunity employer, is operaled by the University of California for the U.S. Department of Energy under contract W-7405-ENG-36. By acceptance of this articte, the publisher recognizes that the U.S. Government relains a nonexclusive, royaltyfree license to publish or reproduce the published form of this contribution, or to allow others to do so, for U.S. Government purposes. The Los Alamos National Laboralory requesis that the publisher identify this article as work performed under the auspices of the U.S. Department of Energy. 


\title{
Safe Extension of Red Blood Cell Storage Life at $4^{\circ} \mathrm{C}$
}

\author{
Mark Bitensky* and Tatsuro Yoshida
}

\begin{abstract}
This is the final report of a one-year, Laboratory-Directed Research and Development (LDRD) project at the Los Alamos National Laboratory (LANL). The project sought to develop methods to extend the storage life of red blood cells. Extended storage would allow donor to self or autologous transfusion, expand and stabilize the blood supply, reduce the costs of medical care and eliminate the risk of transfusion related infections, including a spectrum of hepatitides (A, B \& C) and HIV. The putative cause of red blood cell spoilage at $4^{\circ} \mathrm{C}$ has been identified as oxidative membrane damage resulting from deoxyhemoglobin and its denaturation products including hemichrome, hemin and $\mathrm{Fe}^{3+}$. Trials with carbon monoxide, which is a stabilizer of hemoglobin, have produced striking improvement of red blood cell diagnostics for cells stored at $4^{\circ} \mathrm{C}$. Carbonmonoxy hemoglobin is readily converted to oxyhemoglobin by light in the presence of oxygen. These findings have generated a working model and an approach to identify the best protocols for optimal red cell storage and hemoglobin regeneration.
\end{abstract}

\section{Background and Research Objectives}

1.1 The need for an extended storage life for human red blood cells

There is a compelling international need to develop technology for safely extending the storage life of red blood cells. This need is based in part upon the advantages that result from the practice of autologous transfusion (patient to self) in elective surgery. Autologous donors cannot set aside blood for storage more often than once every ten days without severely depleting their body's red blood cell reserves. Because blood can only be stored safely for 21 days, by the time a patient could donate a third unit, the first unit would already have begun to deteriorate. So, one may only safely donate 2-3 units of blood to oneself, although many current surgical procedures call for 10 or even 15 units! In addition, because of seasonal trends in blood donation, there are periodic blood crises resulting in the delay of urgently

* Principal investigator, FAX: (505) 665-1464 
needed surgical procedures (e.g., valve replacement in a failing heart). Our nation's bloodsupply is thus an undersized, perishable resource that also carries the statistically small but terrible risk of transmitting catastrophic infections or provoking debilitating immunological reactions.

\subsection{Current blood transfusion practice}

Although the transfusion of human blood for elective surgery and trauma has been practiced for almost 200 years, it is only in recent decades that the hazards of transfusion have become more fully recognized. We now understand that transfusions provoke immunological responses: the recipient may reject the transfused cells, or more seriously, the transfused cells can immunologically reject the recipient. In addition, transfusions can introduce viral pathogens including HIV and an alphabet soup of hepatotrophic viruses. Close to 10 billion dollars is spent each year in this country alone for collecting, screening, immunologically matching, and otherwise preparing, scrutinizing and storing red blood cells for transfusion. The national average cost per unit of blood is $\$ 220$ with well over half the cost arising from screening procedures. With current storage practices, after three weeks at $4^{\circ} \mathrm{C}$, blood is properly considered unusable and must be discarded as toxic waste, resulting in the loss of more than 2 million units of blood each year in the USA.

\subsection{Alternatives to current storage practice}

The available alternatives to refrigerated storage are all empirical and neither functionally nor economically viable. The method that comes closest, but is only rarely used, involves freezing red cells and follows practices established in the storage of human and bovine sperm. Costs approach $\$ 1,000$ per unit (not including the cost of maintenance at $-80^{\circ} \mathrm{C}$.) Freezing with glycerol, glycerol removal and the preparation of red cells for transfusion are complex, labor intensive, involve loss of up to 25 percent of the cells and require expensive and complicated instrumentation. Thus, the freezing method is used infrequently, only for the storage of exceedingly rare red blood types. Thus, the other alternatives include the use of liposome encapsulated hemoglobins, cross-linked hemoglobins, and freeze dried red cells. None of these techniques is, at present, even marginally successful.

\subsection{The cause of red blood cell spoilage and a practicable approach}

There is a compelling need to understand $a b$ initio the reasons for red blood cell spoilage at $4^{\circ} \mathrm{C}$. By identifying specific mechanisms responsible for red cell lysis, one can more readily devise a reasoned and practicable method for red cell storage without deterioration. We have identified a probable cause of red cell damage that appears to result from the byproducts of hemoglobin denaturation, hemichrome, and free hemin. The putative identification of hemichrome and hemin as membrane-damaging agents is based on the fact that stabilizing hemoglobin, either with azide or carbon monoxide (Fig. 1), prevents their 
formation, and significantly postpones lysis of red cells stored at $4^{\circ} \mathrm{C}$. Hemichrome is known specifically to cause polymerization of the red cell anion channel (band 3 ) in vivo, resulting in disruption of the red cell membrane cytoskeleton. Hemin acts as a catalyst for oxidative damage to hemoglobin, the membrane cytoskeleton, and the lipid bilayer. Our data do not yet allow us to decide whether damage to the red cell membrane by oxygen radicals, cytoskeletal disruption, or both provide the principal source of red blood cell damage at $4^{\circ} \mathrm{C}$.

We have made a very solid beginning in our efforts to prevent red blood cell deterioration. We have retained between 93-100 percent viable red blood cells in our samples for 95 days by adding azide $\pm \mathrm{Mg}^{2}+$ and refrigerating at $4^{\circ} \mathrm{C}$. This compares with a loss of approximately 50 percent of red cells by 21 to 28 days in the absence of hemoglobin stabilizers (Fig. 2).

\subsection{Benefits of the proposed technology}

The ability to successfully store blood at $4^{\circ} \mathrm{C}$ without deterioration for 150 to 200 days would allow every potential candidate for elective surgery to serve as their own blood donor. This would eliminate the costs of pathogen screening, serological typing, and cross-matching. It would also prevent graft vs. host disease (rejection of the recipient), and eliminate the potential risk of transfusion-borne infection. Even though the risk of viral infection is reduced by screening, it remains real and the penalty is extraordinary! Autologous or patient-to-self transfusion is clearly desirable. In addition, prolonged survival of refrigerated red blood cells would create a more stable and vastly expanded national blood supply. It would also allow the military to reliably stockpile blood in preparation for an emergency. It would markedly reduce the cost of transfusion and diminish the spread of viral infections including HIV. In addition, stable red blood cells would become an effective intravenous platform for the gradual delivery of pharmaceuticals including antibiotics, cancer chemotherapeutic agents, and even protectants against environmental toxins.

\subsection{Project goals}

The goal of this project was to define more precisely the molecular mechanisms of damage by such hemoglobin denaturation products as hemichrome and hemin (a potent oxygen radical generator). We sought to identify and validate other hemoglobin protectants, to extend proof of principle for our hemoglobin damage model and also to test promising hemoglobin stabilization agents. These agents include nitric oxide and cyanide in addition to $\mathrm{CO}$ and azide, both now in laboratory trials. Oxygen appears less attractive, because of its relatively weak affinity for hemoglobin as well as its capacity to enhance methemoglobin formation.

We sought to define the smallest concentrations of azide and cyanide that can effectively prolong red cell refrigerator life. We also sought to define conditions necessary to saturate hemoglobin pools with either $\mathrm{CO}$ or NO. We have used five parameters to assess red cell 
viability. $\mathrm{CO}$ alone, even at less than saturating concentrations, has already shown evidence of excellent hemoglobin stabilization (Fig. 3).

We sought to perfect techniques for totally removing $\mathrm{CO}, \mathrm{NO}$, or other candidate protectants from red cells. In the case of $\mathrm{CO}$, photolysis shows excellent efficiency (at wavelengths between 260 and 480 nanometers) and the purging of $\mathrm{CO}$ is expedited by the presence of a saturating concentration of oxygen.

\section{Importance to LANL's Science and Technology Base and National R\&D Needs}

The project addresses major national and international problems such as the costs of medical care and the risk of transfusion-related hepatitis and AIDS. The studies have important applications in combat casualty care and for preplanned surgery, since they will facilitate the use of autologous transfusion. The project demonstrates the efficacy of the Laboratory's multidisciplinary research approach and the capacity of Los Alamos to find effective solutions and critical technologies for national needs. It utilizes strong Laboratory research capabilities in biophysics, biochemistry, and cell biology and supports a LANL core competency in bioscience and biotechnology.

\section{Scientific Approach and Results to Date}

We routinely obtain red blood cells from a population of more than 40 volunteers, all of whom have given informed consent. Each sample is aliquoted in over 200 tubes, sealed, and kept cold and dark in the presence of one or more of the hemoglobin protectant agents. Thus, testing procedures do not disturb stored blood!

\subsection{What is the mechanism of red cell damage?}

The success of hemoglobin stabilizers in prolonging red cell storage points to hemoglobin denaturation byproducts, hemichromes and hemin, as the destructive agents. We measure hemichrome formation and its association with band 3 during storage by measuring band 3 polymerization using immunofluorescence techniques with band 3 antibodies. We measure the binding of purified labeled autologous antibodies, to stored cells which binding results from band 3 polymerization. And finally, we measure the amount of detergent insoluble aggregates formed by hemichrome band 3 aggregate.

With regard to hemin-induced oxidation of the red cell membrane and other structures, we measure changes in hemin concentrations by extracting hemin and quantitating the procedure spectroscopically. We measure the degree of lipid peroxidation during storage by 
measuring malonaldehyde formation using the thiobarbituric acid assay. We measure protein oxidation during storage assays by measuring the carbonyl content of red cell proteins. All of the parameters mentioned above are measured in protected and unprotected red cells during periods of different storage durations to find correlations that show whether hemichrome formation or hemin-induced peroxidation are predictors of red cell lysis during storage.

3.2 What are the most reliable diagnostic parameters for evaluating red cell viability during storage?

Presently we are using five diagnostic criteria to assess red cells during storage. These include: 1) density gradient centrifugation in Stractan gradients, 2) red cell sodium pump activity, 3) osmotic fragility, 4) hematocrit, and 5) intactness of the cell membrane with vital dyes. A derivative test follows the movement of red cells in capillary surrogate chambers which assesses cell deformability under conditions of variable flow rates. These criteria have strong credentials with regard to the prediction of red cell survival in a transfusion recipient. The most fully studied diagnostic is the Stractan buoyant density gradient. It has been documented repeatedly that less dense cells (which are known to be younger) have extended survivals (multiple weeks) in transfusion recipients. On the other hand, the densest cells (which are known to be presenescent) have shorter survival times (less than one week) in transfusion recipients. Moreover, the osmotic fragility and sodium pump values correlate with gradient position. The younger cells show extremely robust sodium pump activities and reduced osmotic fragility. The older cells (from the bottom of the gradient) show lower sodium pump activities and increased osmotic fragilities. There is also a good correlation with permeability to vital dyes with younger cells being impermeable. Because these criteria are so powerful we use all of them in following the condition of our stored red blood cells. We test all of the above criteria periodically as our storage protocols unfold over time to verify that the quality of protection is solid across the entire diagnostic profile. While the hematocrit is the most convenient indicator of survival it does not have the prognostic power that resides in the rest of the diagnostic profile.

\subsection{How can we identify the most effective protectant?}

Here we have two classes of candidate protectants, nitric oxide and carbon monoxide, which stabilize hemoglobin, while azide and cyanide stabilize methemoglobin. The most promising clinical approach is to use the stabilizers of hemoglobin because of better removability. However, carrying out protection for the longest possible time with methemoglobin stabilizers also has powerful basic implications and helps us to better understand both the mechanisms of damage in refrigerated storage and mechanisms of protection. 
We determine the critical concentration for each of the protectants in order to reach optimal levels of protection. In the case of carbon monoxide and nitric oxide, we find that total saturation is necessary for optimal results. However, in the case of methemoglobin stabilizers, it is possible that smaller concentrations will be effective inasmuch as it is necessary here only to sequester that percentage of hemoglobin that will be converted to methemoglobin during storage. It is not possible to estimate the rate of methemoglobin formation at $4^{\circ} \mathrm{C}$, but we directly measure this rate during the course of our study on the relative efficacy of hemoglobin stabilizers.

3.4 How long can protection be carried out?

This question depends upon our procedures to identify the most effective storage conditions, i.e., which is the best technique for stabilizing hemoglobin. Once the most solid protection format using all diagnostic parameters is ascertained, more extended storage periods are investigated. Because the refrigerator environment is free of turbulance, shear forces, and unstable hemoglobin forms (with the help of protectants), extended shelf life is a perfectly reasonable goal.

3.5 What is the most effective means for removal of the protectants?

This question subdivides into two components. With respect to methemoglobin stabilizing agents such as cyanide and azide, removal is not an experimental goal. These protectants are primarily being utilized for the purpose of mechanistic insight and for the purpose of understanding the potential for prolonged storage. We have no intention of using these compounds clinically. However, the clinical use of nitric oxide or carbon monoxide is attractive provided removal is efficient. Carbon monoxide is clearly the most eminently removable inasmuch as photolysis demonstrates high efficiency (quantum yield of 0.4 ) and offers striking advantages. If carbon monoxide is able to provide adequate protection, it will be the protectant of choice. On the other hand, if nitric oxide is needed, the possibility exists that NO may be purged by flushing with carbon monoxide and subsequently removing carbon monoxide by photolysis.

3.6 What is the extent and source of differences from subject to subject?

We have observed that levels of protection can vary from subject to subject even though the buffers, protectants, and conditions for collecting and storing blood are identical. We attempt to document the extent of differences in order to understand the efficacy of hemoglobin stabilization programs over broad subject populations. We also attempt to gather information about the metabolic/genetic basis of differences including differences in the levels of methemoglobin, reduced glutathione, and possibly different hemoglobin types, some of which are known to be more unstable. 


\subsection{Results to date}

These studies in red blood cell storage have already achieved striking results that have established the principal of extended storage. Our studies with human erythrocytes have resulted in the elucidation of biochemical explanations for specific pathogenetic changes that befall the red cell during prolonged storage in the refrigerator $\left(4^{\circ} \mathrm{C}\right)$. As a consequence of these studies, we have learned that it is essential to stabilize or protect hemoglobin against oxidative damage, which is paradoxically increased in the cold. These experimental insights have facilitated the development of a new red cell storage protocol. The resulting technology has already provided a doubling in refrigerated storage times as well as striking improvement in the quality of the stored red cells.

\section{DISCLAIMER}

account of work sponsored by an agency of the United States United States Government nor any agency thereof, nor any of their Government. Neither the United States or implied, or assumes any legal liability or product, or employees, makes any warranty, exps, or usefulness of any information, appanned rights. Referbility for the accuracy, complets that its use would not infringe privately owed rige, trademark, process disclosed, or represents thercial product, process, or service by trs endorsement, recomence herein to any specific com not necessarily constitute or imply its endoren. The views manufacturer, or otherwise doe United States Government or any agency then those of the mendation, or favoring by the United herein do not and opinions of authors express agency thereof.

United States 


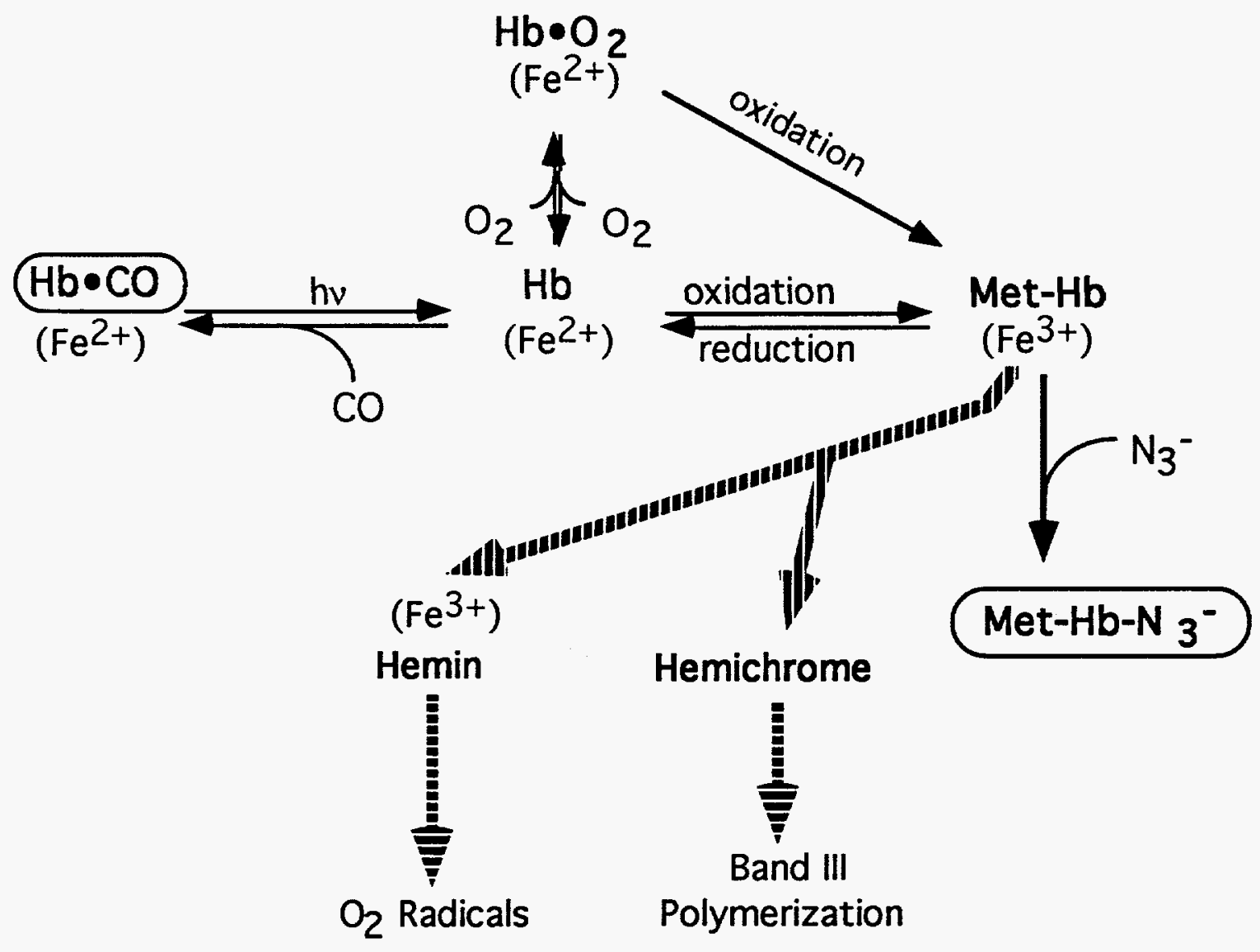

Figure 1. Figure shows stabilization of methemoglobin by azide and stabilization of hemoglobin by carbon monoxide. 


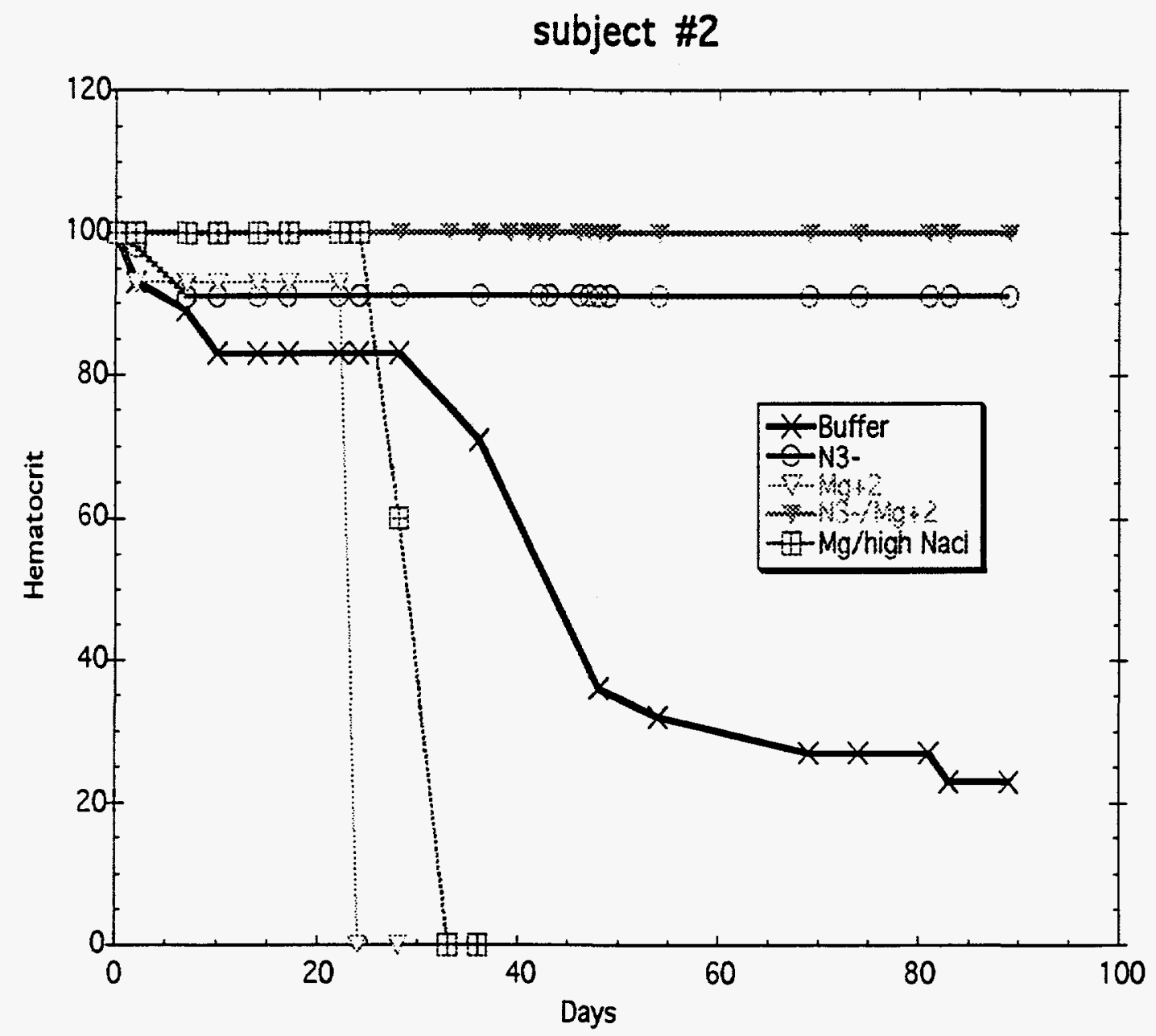

Figure 2. Figure shows excellent protection for more than 90 days with $4 \mathrm{mM}$ sodium azide with/without $\mathrm{Mg}^{2+}$ (the two upper curves). The three falling curves represent samples stored with either $\mathrm{Mg}^{2+}$ alone, $\mathrm{Mg}^{2+}$ and $\mathrm{NaCl}$, or neither in standard blood banking buffer. 


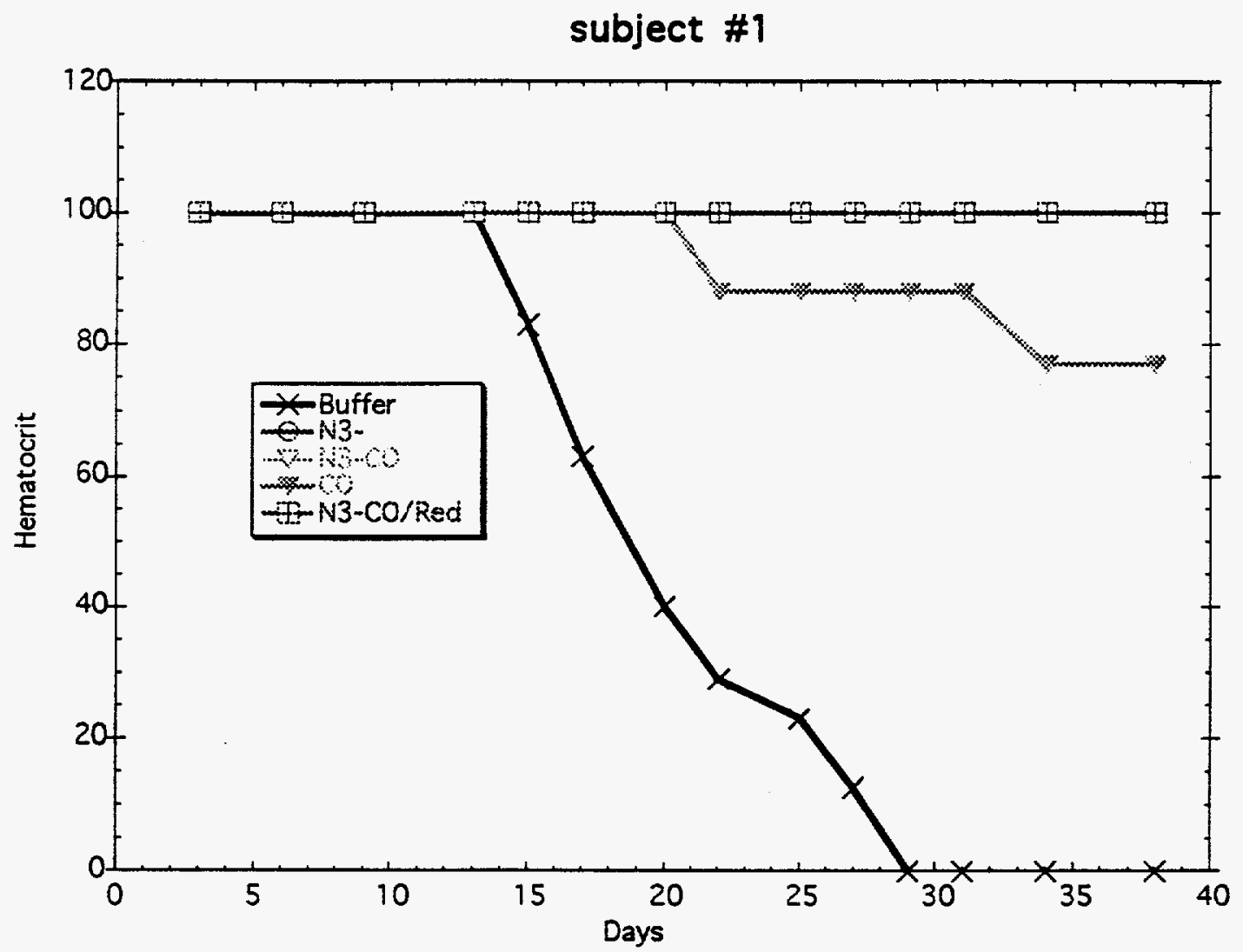

Figure 3. This shows rapid spoilage of unprotected red cells (the steeply falling line) and extended protection by unsaturating concentrations of carbon monoxide (second line from the top) and carbon monoxide with azide giving full protection for more than 38 days (top line). 